\title{
Curvature Based Biomarkers for Diabetic Retinopathy via Exponential Curve Fits in SE(2)
}

\author{
Erik J. Bekkers ${ }^{1}$, Jiong Zhang ${ }^{1}$, Remco Duits ${ }^{1}$, and Bart M. ter Haar \\ Romeny ${ }^{2,1}$ \\ 1 Department of Biomedical Engineering and Department of Mathematics and \\ Computer Science, Eindhoven University of Technology, the Netherlands \\ 2 Department of Biomedical and Information Engineering, \\ Northeastern University, Shenyang, China \\ \{e.j.bekkers, j.zhang1, r.duits, b.m.terhaarromeny\}@tue.nl
}

\begin{abstract}
We propose a robust and fully automatic method for the analysis of vessel tortuosity. Our method does not rely on pre-segmentation of vessels, but instead acts directly on retinal image data. The method is based on theory of best-fit exponential curves in the roto-translation group SE(2). We lift 2D images to 3D functions called orientation scores by including an orientation dimension in the domain. In the extended domain of positions and orientations (identified with $\mathrm{SE}(2)$ ) we study exponential curves, whose spatial projections have constant curvature. By locally fitting such curves to data in orientation scores, via our new iterative stabilizing refinement method, we are able to assign to each location a curvature and confidence value. These values are then used to define global tortuosity measures. The method is validated on synthetic and retinal images. We show that the tortuosity measures can serve as effective biomarkers for diabetes and different stages of diabetic retinopathy.
\end{abstract}

Keywords: Retina, vessel tortuosity, biomarkers, diabetes, curvature, orientation scores, roto-translation group

\section{Introduction}

Systemic diseases, such as diabetes, may cause quantifiable changes to the geometry of the retinal microvasculature $[1,2]$. One of the most relevant geometrical features of the microvasculature is vessel tortuosity [2-5]. While for some geometrical features (such as vessel calibre) there is a general consensus [1] on how they are associated to several diseases. This is not the case for vessel tortuosity, which makes it still a very relevant topic of research. E.g., in [3] a positive, and in [4] a negative association of vessel tortuosity with progression towards diabetic retinopathy (DR) is found. In this work, we present a novel robust and fully automated method for the extraction of tortuosity measures, and show strong positive associations of the measures with diabetes and progressive stages of DR.

Vessel tortuosity descriptors are typically computed via an extensive pipeline (including manual interventions) of image pre-processing, segmentation, thinning

X. Chen, M. K. Garvin, J. Liu, E. Trucco, Y. Xu (Eds.): OMIA 2015, Held in Conjunction with MICCAI 2015, Munich, Germany, Iowa Research Online, pp. 113-120, 2015. Available from: http://ir.uiowa.edu/omia/2015_Proceedings/2015/ 
and splitting of the vascular network, after which tortuosity values are computed from the extracted vessel centerlines $[2,3,5,6]$. In such pipelines, errors introduced in each processing step may accumulate, and information might get lost along the way. As an alternative, we propose a reduced pipeline that does not rely on explicit segmentation of the blood vessels, but instead computes tortuosity features directly from retinal image data.

The proposed method is based on theory of best exponential curve fits in the roto-translation group $S E(2)$, developed by Duits, Franken and Janssen [7$9]$. To this end, we lift $2 \mathrm{D}$ images to $3 \mathrm{D}$ functions called orientation scores by including an orientation dimension [10]. In the extended domain of positions and orientations (identified with $S E(2)$ ) we study so-called exponential curves, whose curvatures are constant. By locally fitting exponential curves [8] to data in orientation scores, we are able to assign to each location a curvature and measurement-confidence value, which we use to define global tortuosity measures. Additionally, we improve the accuracy of best-exponential curve fits via a novel refinement procedure, resulting in more accurate curvature estimations.

This article is structured as follows: Sec. 2 provides necessary prerequisites: the notion of curves in the space of positions and orientations $\mathbb{R}^{2} \rtimes S^{1}$ (Subsec. 2.1), theory on orientation scores (functions on this space) and on the computation of best-exponential curve fits from these functions (Subsec. 2.2). In Subsec. 2.3 we then introduce our tortuosity measures based on the aforementioned theory. In Sec. 3 we validate the accuracy of the curvature extraction and associated confidence, and demonstrate the potential of using our tortuosity measures as biomarkers in diabetes research. The article is concluded in Sec. 4.

A

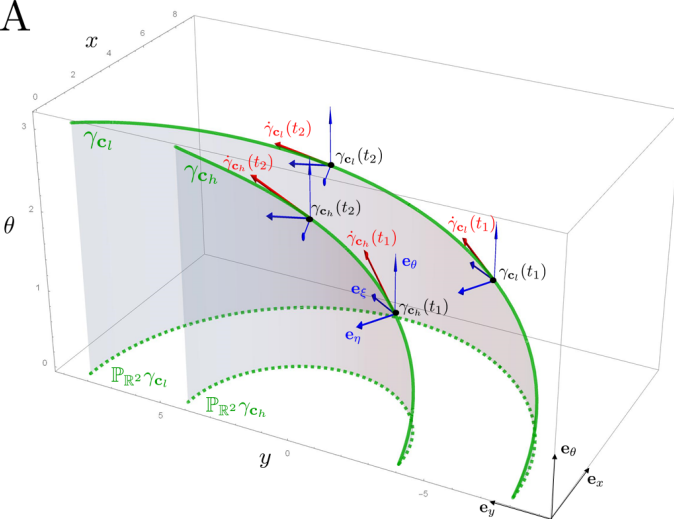

B low curvature

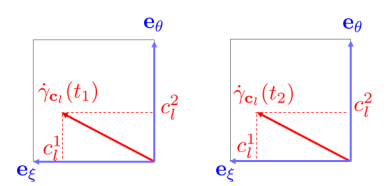

C high curvature

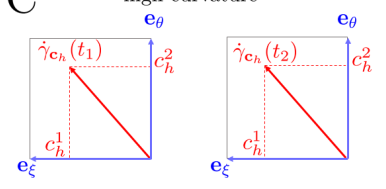

Fig. 1: A: Two exponential curves $\gamma_{\mathbf{c}_{h}}$ and $\gamma_{\mathbf{c}_{l}}$ in $S E(2)$, with high and low curvature respectively. The coefficients $\mathbf{c}=\left(c^{\xi}, c^{\eta}, c^{\theta}\right)^{T}$ of tangent vectors $\dot{\gamma}_{\mathbf{c}}(t)$, expressed in the left invariant basis at location $\gamma_{\mathbf{c}}(t)$ are constant along the exponential curves $\gamma_{\mathbf{c}}$. This is emphasized in $\mathbf{B}$ and $\mathbf{C}$, where one also observes a steeper slope in $\theta$-direction of the tangent vector $\dot{\gamma}_{\mathbf{c}_{h}}$ compared to $\dot{\gamma}_{\mathbf{c}_{l}}$. 


\section{Theory}

\subsection{Exponential Curves in $\mathrm{SE}(2)$}

The domain $\mathbb{R}^{2} \rtimes S^{1} \equiv S E(2)$. The joint space of positions and orientations, with elements $(\mathbf{x}, \theta) \in \mathbb{R}^{2} \rtimes S^{1}$, is essentially the roto-translation group $\mathrm{SE}(2)$ of planar translations and rotations, equipped with group product $g \cdot g^{\prime}=(\mathbf{x}, \theta) \cdot\left(\mathbf{x}^{\prime}, \theta^{\prime}\right)=$ $\left(R_{\theta} \mathbf{x}^{\prime}+\mathbf{x}, \theta+\theta^{\prime}\right)[9$, ch. 2.1].

Curves and tangent vectors in $S E(2)$. Planar curves $\gamma_{2 D}(t)=(x(t), y(t))^{T} \in$ $\mathbb{R}^{2}$ can be naturally lifted to curves $\gamma_{S E(2)}(t)=(x(t), y(t), \theta(t))^{T} \in S E(2)$ in the space of positions and orientations by considering the direction of the tangent vector $\dot{\gamma}_{2 D}(t)$ as the third coordinate $(\theta(t)=\arg (\dot{x}(t)+i \dot{y}(t)))$. Tangent vectors of planar curves $\dot{\gamma}(t)=(\dot{x}(t), \dot{y}(t)) \in T\left(\mathbb{R}^{2}\right)$ are usually spanned by a global basis $\left\{\mathbf{e}_{x}, \mathbf{e}_{y}\right\}$, with $\mathbf{e}_{x}=(1,0)$ and $\mathbf{e}_{y}=(0,1)$, i.e., $T\left(\mathbb{R}^{2}\right)=\operatorname{span}\left\{\mathbf{e}_{x}, \mathbf{e}_{y}\right\}$. In $S E(2)$ we must work with a rotating frame of reference $\left\{\mathbf{e}_{\xi}(g), \mathbf{e}_{\eta}(g), \mathbf{e}_{\theta}(g)\right\}=$ $\left\{\cos \theta \mathbf{e}_{x}+\sin \theta \mathbf{e}_{y},-\sin \theta \mathbf{e}_{x}+\cos \theta \mathbf{e}_{y}, \mathbf{e}_{\theta}\right\}$, aligned with the orientation at each $g \in$ $S E(2)$, rather than with a global frame $\left\{\mathbf{e}_{x}, \mathbf{e}_{y}, \mathbf{e}_{\theta}\right\}$. The tangent space at each $g$ is spanned by the left-invariant frame $T_{g}(S E(2))=\operatorname{span}\left\{\mathbf{e}_{\xi}(g), \mathbf{e}_{\eta}(g), \mathbf{e}_{\theta}(g)\right\}$.

Exponential curves in $S E(2)$. An exponential curve is a curve whose tangent vector components $\mathbf{c}=\left(c^{\xi}, c^{\eta}, c^{\theta}\right)^{T}$ expressed in the local left-invariant basis $\left.\left\{\mathbf{e}_{\xi}, \mathbf{e}_{\eta}, \mathbf{e}_{\theta}\right\}\right|_{\gamma_{\mathbf{c}}}$ are constant, i.e., $\dot{\gamma}_{\mathbf{c}}(t)=c^{\xi} \mathbf{e}_{\xi}\left(\gamma_{\mathbf{c}}(t)\right)+c^{\eta} \mathbf{e}_{\eta}\left(\gamma_{\mathbf{c}}(t)\right)+c^{\theta} \mathbf{e}_{\theta}\left(\gamma_{\mathbf{c}}(t)\right)$, for all $t \in \mathbb{R}$. Exponential curves in $S E(2)$ can be regarded as "straight lines" with respect to the curved geometry of $S E(2)$. The exponential curve through $g$ with tangent $\mathbf{c}$ is given by $\gamma_{\mathbf{c}}^{g}(t)=g \cdot \exp \left(t\left(\sum_{i=1}^{3} c^{i} A_{i}\right)\right)$ with $\left\{A_{1}, A_{2}, A_{3}\right\}=$ $\left\{\mathbf{e}_{\xi}(\mathbf{0}, 0), \mathbf{e}_{\eta}(\mathbf{0}, 0), \mathbf{e}_{\theta}(\mathbf{0}, 0)\right\}$ the basis for the Lie algebra. By direct computation it follows that $\gamma_{\mathbf{c}}^{g}$ is a helix with constant curvature and torsion in $S E(2)$. For details see $[11,8]$. For intuition see Fig. 1. The explicit formulas for these exponential curves are well-known (see e.g. $[12,8]$ ). Here however, we do not need these formulas as we directly deduce curvature of spatially projected curves $\mathbb{P}_{\mathbb{R}^{2}} \gamma_{\mathbf{c}}$ (cf. Fig. 1) from vector c via

$$
\kappa=\frac{c^{\theta} \operatorname{sign}\left(c^{\xi}\right)}{\sqrt{\left|c^{\xi}\right|^{2}+\left|c^{\eta}\right|^{2}}} .
$$

See also [9, ch. 2.9] for more details.

\subsection{Image Data Analyzed as Function on SE(2)}

Orientation scores. We analyse image data in the form of orientation scores, which are functions on $S E(2)$. An orientation score $U$ can be constructed from an image $f$ by means of correlation with some anisotropic wavelet $\psi$ via

$$
U(\mathbf{x}, \theta)=\left(\mathcal{W}_{\psi} f\right)(\mathbf{x}, \theta)=\left(\bar{\psi}_{\theta} \star f\right)(\mathbf{x})=\int_{\mathbb{R}^{2}} \overline{\psi\left(\mathbf{R}_{\theta}^{-1}(\tilde{\mathbf{x}}-\mathbf{x})\right)} f(\tilde{\mathbf{x}}) d \tilde{\mathbf{x}},
$$

where $\psi \in \mathbb{L}_{2}\left(\mathbb{R}^{2}\right)$ is the correlation kernel, aligned with the $x$-axis, where $\mathcal{W}_{\psi}$ denotes the transformation between image $f$ and orientation score $U$, and $\star$ denotes correlation. The overline denotes complex conjugation, $\psi_{\theta}(\mathbf{x})=\psi\left(\mathbf{R}_{\theta}^{-1} \mathbf{x}\right)$ 


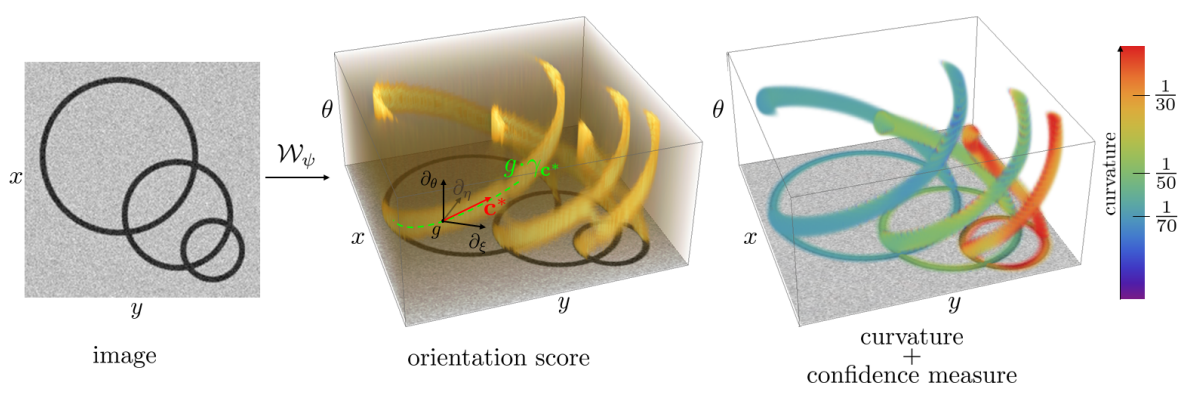

Fig. 2: Construction of an orientation score (OS) (middle panel) from an image (left panel) via the OS-transform $\mathcal{W}_{\psi}$. In the score a derivative frame $\left\{\partial_{\xi}, \partial_{\eta}, \partial_{\theta}\right\}$, aligned with group elements $(x, y, \theta)$, is used for tangent vector $\left(\mathbf{c}^{*}\right)$ estimation of exponential curves $\gamma_{\mathbf{c}^{*}}$. Using $\mathbf{c}^{*}$, curvature and measurement confidence values can be computed, which are encoded resp. in color and opacity in the right panel.

and $\mathbf{R}_{\theta}$ is a counter clockwise rotation over angle $\theta$. We choose cake wavelets [10] for $\psi$. The Fourier transforms of cake wavelets uniformly cover the frequency domain, and have thereby the advantage over other oriented wavelets (e.g. Gabor wavelets) that they allow for a stable inverse transformation $\mathcal{W}_{\psi}^{*}$ from the orientation score back to the image. As such, they ensure that no data-evidence is lost in the transformation. The left two panels of Fig. 2 show an image with different curvature circles and the corresponding orientation score.

Best exponential curve fits. We compute curvature values directly from tangent vectors (see Eq. (1)) of exponential curves that locally best fit the data. In medical image analysis applications the direction of minimal principal curvature, obtained via eigensystem analysis of the Hessian matrix, is often used in the computation of vectors tangent to oriented (tubular) structures. This concept is for example used in the Frangi vesselness filter [13]. Here we exploit a similar approach, however, when considering the curved domain $\mathbb{R}^{2} \rtimes S^{1}$ we must pay attention to the following:

1. Rather than using a global $\left\{\partial_{x}, \partial_{y}, \partial_{\theta}\right\}$ derivative frame (we use short hand notation $\partial_{i}=\frac{\partial}{\partial i}$ ) we must take into consideration the curved geometry of the domain, and compute the Hessian matrix via left-invariant derivatives:

$$
\mathcal{H} U=\mathbf{M}_{\mu^{-2}}\left(\begin{array}{ccc}
\partial_{\xi}^{2} U & \partial_{\xi} \partial_{\eta} U & \partial_{\theta} \partial_{\xi} U \\
\partial_{\xi} \partial_{\eta} U & \partial_{\eta}^{2} U & \partial_{\theta} \partial_{\eta} U \\
\partial_{\xi} \partial_{\theta} U & \partial_{\eta} \partial_{\theta} U & \partial_{\theta}^{2} U
\end{array}\right) \mathbf{M}_{\mu^{-2}}
$$

with $\left\{\partial_{\xi}, \partial_{\eta}, \partial_{\theta}\right\}=\left\{\cos \theta \partial_{x}+\sin \theta \partial_{y},-\sin \theta \partial_{x}+\cos \theta \partial_{y}, \partial_{\theta}\right\}$, and with $\mathbf{M}_{\mu}=\operatorname{diag}(\mu, \mu, 1)$. Here parameter $\mu$, with unit $1 /$ length, is introduced to deal with the different physical dimensions in domain $\mathbb{R}^{2} \rtimes S^{1}[8,9]$.

2. Since left-invariant derivatives are non-commutative, e.g. $\partial_{\theta} \partial_{\xi} U \neq \partial_{\xi} \partial_{\theta} U$, the Hessian matrix $\mathcal{H} U$ is not symmetric. In order to obtain real-valued eigenvalues of a dimensionless matrix, we symmetrize the Hessian matrix via $\mathcal{H}_{\mu} U=\mathbf{M}_{\mu}(\mathcal{H} U)^{T} \mathbf{M}_{\mu^{2}}(\mathcal{H} U) \mathbf{M}_{\mu}$, and perform eigenanalysis on $\mathcal{H}_{\mu} U$. 
Eigenvector $M_{\mu} \mathbf{c}^{*}$ with lowest eigenvalue of the dimensionless ( $\mu$-scaled) matrix $\mathcal{H}_{\mu} U$ does not give the minimal principal curvature direction, but rather provides the solution to the following optimization problem [8]:

$$
\mathbf{c}^{*}(g)=\arg \min _{\mathbf{c} \in \mathbb{R}^{3},\|\mathbf{c}\|_{\mu}=1}\left\{\left\|\left.\frac{d}{d t}\left(\nabla U\left(\gamma_{\mathbf{c}}^{g}(t)\right)\right)\right|_{t=0}\right\|_{\mu}^{2}\right\},
$$

with left-invariant gradient $\nabla U=\mathbf{M}_{\mu^{-2}}\left(\partial_{\xi} U, \partial_{\eta} U, \partial_{\theta} U\right)^{T}$ and $\|\mathbf{c}\|_{\mu}^{2}=\mu^{2}\left|c^{\xi}\right|^{2}+$ $\mu^{2}\left|c^{\eta}\right|^{2}+\left|c^{\theta}\right|^{2}$. Intuitively speaking, $\mathbf{c}^{*}$ gives the tangent vector components of the exponential curve $\gamma_{\mathbf{c}^{*}}^{g}(t)$, starting at position $g$, along which the left-invariant gradient has fewest variations (Fig. 2). Alternatively, in work by van Ginkel [14], tangent estimation was done based on the structure tensor. A full overview of exponential curve fit models, with 3D extensions, can be found in [8].

\subsection{Curvature, Confidence and Global Tortuosity Measures}

In our implementation we use Gaussian derivatives to compute the Hessian matrix, i.e., in Eq. (3) we substitute $U \leftarrow G_{\sigma_{s}, \sigma_{o}} * U$, with $G_{\sigma_{s}, \sigma_{o}}(\mathbf{x}, \theta)$ a Gaussian kernel with spatial isotropic scale $\frac{1}{2} \sigma_{s}^{2}$, and orientation scale $\frac{1}{2} \sigma_{o}^{2}$. For each tangent component $\mathbf{c}^{*}(g)$ of the best exponential curve fit at location $g$, we compute a curvature value $\kappa(g)$ directly via Eq. (1). For each location we also determine a confidence measure $s(g)$ based on blob detection via the Laplacian computed in the plane orthogonal to the tangent direction $\mathbf{c}^{*}(g)$ via

$$
\begin{aligned}
& s(g)=(\mathcal{S}(U))(g)=\left(-\Delta_{o} U(g)\right)_{+}= \\
& \quad\left(-\left(\mathbf{e}_{1}^{o}(g)\right)^{T} \mathbf{M}_{\mu^{2}} \mathcal{H} U(g) \mathbf{M}_{\mu^{2}} \mathbf{e}_{1}^{o}(g)-\left(\mathbf{e}_{2}^{o}(g)\right)^{T} \mathbf{M}_{\mu^{2}} \mathcal{H} U(g) \mathbf{M}_{\mu^{2}} \mathbf{e}_{2}^{o}(g)\right)_{+},
\end{aligned}
$$

where $(v)_{+}=\max \{v, 0\}$, and $\mathbf{M}_{\mu} \mathbf{e}_{1}^{o}(g)$ and $\mathbf{M}_{\mu} \mathbf{e}_{2}^{o}(g)$ are two eigenvectors of $\mathcal{H}_{\mu} U$ orthonormal to $\mathbf{M}_{\mu} \mathbf{c}^{*}(g)$ [9, ch. 5.3]. Improved accuracy of the confidence and curvature measurements is achieved via the following stabilizing refinement scheme:

$$
s_{n+1}=\mathcal{S}\left(s_{n}\right), \text { with } s_{1}=\mathcal{S}(U), \quad \kappa_{n+1}=\mathcal{K}\left(s_{n}\right), \text { with } \kappa_{1}=\mathcal{K}(U),
$$

where we denote the computation of the confidence map $s$ from input volume $U$ (using Equations (3)-(5)) with $\mathcal{S}(U)$, and the computation of curvature $\kappa$ (using Equations $(3),(4)$ and $(1))$ with $(\mathcal{K}(U))(g)=\frac{c(g)^{\theta} \operatorname{sign}\left(c(g)^{\xi}\right)}{\sqrt{\left|c(g)^{\xi}\right|^{2}+\left|c(g)^{\eta}\right|^{2}}}$. From the curvature and measurement confidence functions we compute the following global tortuosity features:

$$
\begin{aligned}
\mu_{|\kappa|} & =\frac{1}{s_{\text {total }}} \int_{-\infty}^{\infty} \int_{0}^{\pi}|\kappa(\mathbf{x}, \theta)| s(\mathbf{x}, \theta) \mathrm{d} \mathbf{x} \mathrm{d} \theta \\
\sigma_{|\kappa|} & =\sqrt{\frac{1}{s_{\text {total }}} \int_{-\infty}^{\infty} \int_{0}^{\pi}\left(|\kappa(\mathbf{x}, \theta)|-\mu_{|\kappa|}\right)^{2} s(\mathbf{x}, \theta) \mathrm{d} \mathbf{x} \mathrm{d} \theta}
\end{aligned}
$$

with $s_{\text {total }}=\int_{-\infty}^{\infty} \int_{0}^{\pi} s(\mathbf{x}, \theta) d \mathbf{x} d \theta$. Features $\mu_{|\kappa|}$ and $\sigma_{|\kappa|}$ give respectively the weighted mean and standard deviation of absolute curvatures. 


\section{Validation and Application to Clinical Data}

The scales $\sigma_{s}=3$ and $\sigma_{o}=\frac{\pi}{18}$ are fixed in all experiments, and are chosen as to best match the cross-sectional scales of vessels in the orientation score (Subsec. 2.3). We set $\mu=\frac{\sigma_{o}}{\sigma_{s}}$, and sampled the orientation score with 18 orientations.

Validation. Our method was validated on two synthetic images (201px by $201 \mathrm{px})$ with Gaussian white noise $(\mathrm{SNR}=1)$ : One image composed of three circles with radii of 50px, 70px and 90px; One image composed of three crossing Euler spirals. The curvatures computed with our method (third and fourth column Fig. 3), with $n=1$ and $n=10$ refinement iterations (Eq. (6)), were compared against the ground truth (second column Fig. 3). In the curvature maps, curvature is encoded with color and confidence with opacity (see e.g. also Fig. 2). Visual comparison shows a remarkable agreement between our method and the ground truth, and we observe improved precision of both the confidence and curvature measurements with an increasing number of refinement iterations $n$. This is also confirmed by the comparison of curvature measurements against the ground truth via scatter plots (most right two figures in Fig. 3). The root mean squared error of $|\kappa|$ was reduced from 0.0138 for $n=1$ to 0.0024 for $n=10$.

Application to clinical data. Tortuosity measures $\mu_{|\kappa|}$ and $\sigma_{|\kappa|}$ were computed on images of two publicly available databases: 1) the high resolution fundus (HRF) database [15], consisting of 15 images of healthy controls, and 15 images of diabetes patients; 2) the MESSIDOR database [16], consisting of 1200 images of diabetes patients which are graded for diabetic retinopathy: R0 (no retinopathy), R1, R2 and R3 (severe retinopathy). All images are made with 45 degree field of view (FOV) cameras, however with varying image resolutions. In order to have approximately the same physical pixel size in all images, they are cropped and resized such that the FOV area spans a width of 1024px. Curvature and confidence measures were computed with $n=3$ refinement iterations.

Fig. 4 shows a selection of results. Fig. 5 and Tab. 1 show the distribution of feature values for different subgroups of the HRF and MESSIDOR database. Based on a Mann-Whitney U test (p-values reported in Tab. 1) we conclude that all subgroups show a significant increase in $\mu_{|\kappa|}$ and $\sigma_{|\kappa|}$ in comparison to the corresponding base groups (healthy for HRF, and R0 for MESSIDOR).

We also observe that our method detects microbleeds and hemorrhages as high curvature regions (Fig. 4). While this was not our intention, it is a very welcome property when using features $\mu_{|\kappa|}$ and $\sigma_{|\kappa|}$ as biomarkers for DR. However, for research dedicated to the retinal vasculature one only wants to analyse blood vessels. We plan to address this feature in future work via the construction of vessel specific confidence measures (e.g. vesselness in orientation scores $[13,8]$ ).

\section{Conclusion}

We developed new vessel tortuosity descriptors based on curvature estimations from best exponential curve fits in orientation scores. Furthermore, a novel refinement scheme was presented for more accurate curvature and confidence mea- 


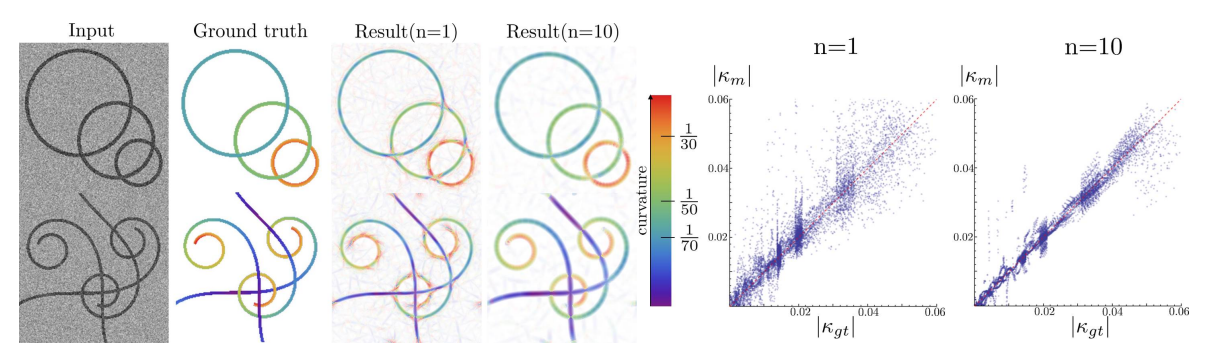

Fig. 3: From left to right: input image $(\mathrm{SNR}=1)$, ground truth color-coded curvature map, measured curvature map with resp. $n=1$ and $n=10$ refinements, scatter plot of ground truth vs measured curvatures for resp. $n=1$ and $n=10$.

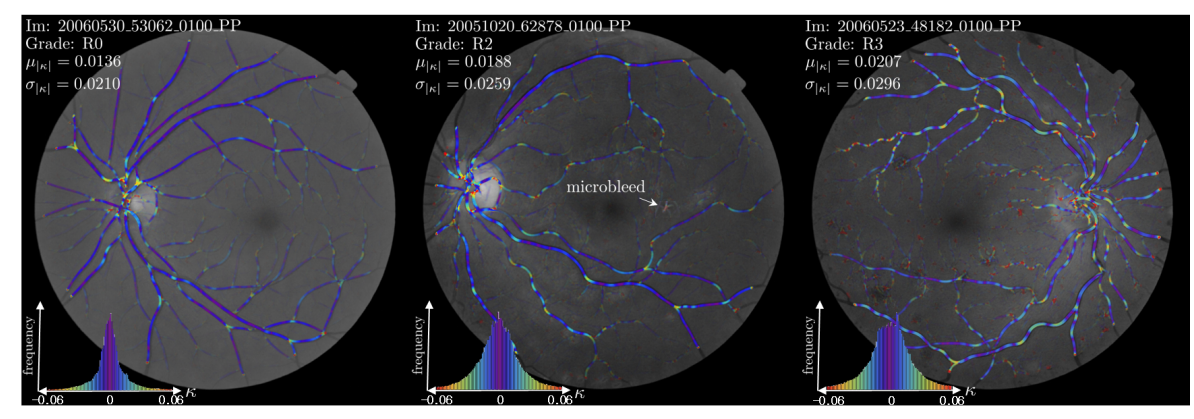

Fig. 4: Results on three images of the MESSIDOR database. Measured absolute curvature $|\kappa|$ encoded in color, and confidence $s$ encoded with opacity, overlain on the original image, together with the histogram of measured $|\kappa|$ values.

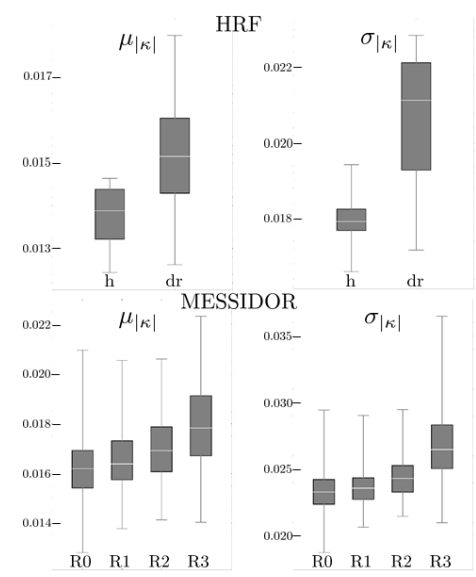

Fig. 5: Box-and-whisker plots of tortuosity measures $\mu_{|\kappa|}$ and $\sigma_{|\kappa|}$ in subgroups of the HRF and MESSIDOR database.
Table 1: Tortuosity measures $\mu_{|\kappa|}$ and $\sigma_{|\kappa|}$ in the HRF and MESSIDOR database.

\begin{tabular}{|c|c|c|}
\hline \multirow[t]{2}{*}{ Subgroup } & \multicolumn{2}{|c|}{ Mean $\pm(\mathrm{STD})$} \\
\hline & $\mu_{|\kappa|}\left(10^{-2}\right)$ & $\sigma_{|\kappa|}\left(10^{-2}\right)$ \\
\hline & $\mathrm{HRF}$ & \\
\hline Healthy & $1.372 \pm(0.069)$ & $1.796 \pm(0.072)$ \\
\hline Diabetic & $1.521 \pm(0.130)$ & $2.073 \pm(0.185)$ \\
\hline p-value ${ }^{a}$ & $<0.001$ & $<0.001$ \\
\hline & - MESSIDOR & \\
\hline R0 & $1.624 \pm(0.120)$ & $2.333 \pm(0.134)$ \\
\hline $\mathrm{R} 1$ & $1.657 \pm(0.124)$ & $2.365 \pm(0.131)$ \\
\hline p-value ${ }^{b}$ & 0.007 & 0.020 \\
\hline $\mathrm{R} 2$ & $1.698 \pm(0.122)$ & $2.436 \pm(0.144)$ \\
\hline $\mathrm{p}$-value ${ }^{b}$ & $<0.001$ & $<0.001$ \\
\hline R3 & $1.795 \pm(0.160)$ & $2.674 \pm(0.235)$ \\
\hline p-value ${ }^{b}$ & $<0.001$ & $<0.001$ \\
\hline
\end{tabular}

${ }^{a}$ Compared to Healthy.

${ }^{b}$ Compared to R0. 
sures. Validation on synthetic images showed high accuracy of our curvature extraction approach. Application to clinical retinal image datasets showed strong positive associations of the proposed tortuosity descriptors with diabetes and different stages of diabetic retinopathy (DR). As such, we see high potential of the method to be used in a screening setting for diabetes and DR detection.

Acknowledgements: This work is part of Hé Programme of Innovation, which is (partly) financed by the Netherlands Organisation for Scientific Research (NWO). The research leading to these results has also received funding from the ERC council under the EC's 7th Framework Programme (FP7/2007-2013) / ERC grant agr. No. 335555.

\section{References}

1. Ikram, M.K., Ong, Y.T., Cheung, C.Y., Wong, T.Y.: Retinal vascular caliber measurements: Clinical significance, current knowledge and future perspectives. Ophthalmologica 229(3) (2013) 125-136

2. Kalitzeos, A.A., Lip, G.Y., Heitmar, R.: Retinal vessel tortuosity measures and their applications. Experimental Eye Research 106(0) (2013) 40 - 46

3. Cheung, C., Lamoureux, E., Ikram, M., et al.: Retinal vascular geometry in Asian persons with diabetes and retinopathy. J Diabetes Sci Technol 6(3) (2012) 595-605

4. Sasongko, M., Wong, T., Nguyen, T., et al.: Retinal vascular tortuosity in persons with diabetes and diabetic retinopathy. Diabetologia 54(9) (2011) 2409-2416

5. Hart, W.E., Goldbaum, M., Kube, P., Nelson, M.R.: Measurement and classification of retinal vascular tortuosity. IJMI 53 (1999) 239-252

6. Wilson, C.M., et al.: Computerized analysis of retinal vessel width and tortuosity in premature infants. nvest. Ophthalmol. Vis. Sci. 49(8) (2008) 3577

7. Duits, R., E.M., F.: Left-invariant parabolic evolutions on $\mathrm{SE}(2)$ and contour enhancement via invertible orientation scores. part II: Non-linear left-invariant diffusions on invertible orientation score. Q APPL MATH 68 (2010) 293-331

8. Duits, R., Janssen, M., Hannink, J., Sanguinetti, G.: Locally adaptive frames in the roto-translation group and their applications in medical imaging. arXiv preprint arXiv:1502.08002 (2015)

9. Franken, E., Duits, R.: Crossing-preserving coherence-enhancing diffusion on invertible orientation scores. IJCV 85(3) (December 2009) 253-278

10. Bekkers, E., Duits, R., Berendschot, T., ter Haar Romeny, B.: A multi-orientation analysis approach to retinal vessel tracking. JMIV (2014) 1-28

11. Chirikjian, G.S.: Engineering Applications of Noncommutative Harmonic Analysis: With Emphasis on Rotation and Motion Groups. CRC Press, Abingdon (2000)

12. Sanguinetti, G., Citti, G., Sarti, A.: A model of natural image edge co-occurrence in the rototranslation group. Journal of Vision 10(14) (December 2010)

13. Frangi, A., Niessen, W., Vincken, K., Viergever, M.: Multiscale vessel enhancement filtering. In Wells, W., Colchester, A., Delp, S., eds.: MICCAI98. Volume 1496 of LNCS. Springer Berlin Heidelberg (1998) 130-137

14. van Ginkel, M.: Image Analysis using Orientation Space based on Steerable Filters. PhD thesis, Technical University Delft (2002)

15. Odstrcilik, J., et al.: Retinal vessel segmentation by improved matched filtering: evaluation on a new high-resolution fundus image database. IEEE TIP 7 (June 2013) 373-383(10)

16. MESSIDOR: Methods to evaluate segmentation and indexing techniques in the field of retinal ophthalmology. http://messidor.crihan.fr/download-en.php 